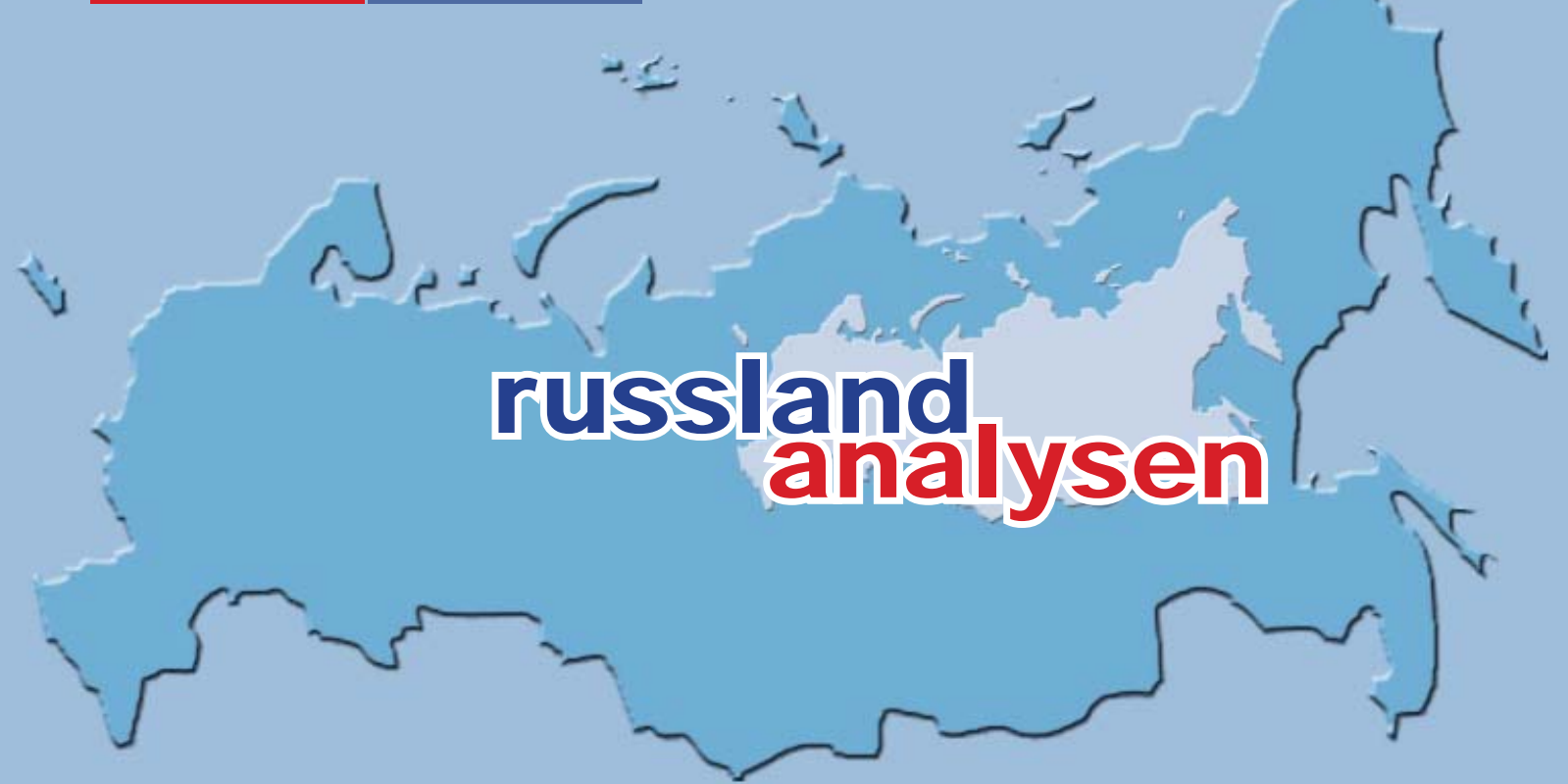

\title{
russlandanalysen.de
}

\section{DER DUMAWAHLKAMPF 2007}

\section{ANALYSE}

Dumawahlkampf 2007

Jens Siegert, Moskau

TABELLEN UND GRAFIKEN ZUM TEXT

Welche folgender Parteien würden Sie bei den Wahlen für die Staatsduma Russlands wählen, wenn sie nächsten Sonntag stattfinden würden?

Diskussionen über die Wahlen

Im Vorfeld der Wahlen ...

DOKUMENTATION

Die »Junge Garde von `Einiges Russland« in Barnaul verunglimpft Portraits von

Oppositionellen

KOMMENTAR

Nachfolgeproblem und Dumawahl

Jens Siegert, Moskau

DOKUMENTATION

Das ODIHR sieht sich nicht in der Lage, die russischen Dumawahlen zu beobachten

PRESSESTIMMEN

Pressestimmen zur Absage der Wahlbeobachtung durch das ODIHR

\section{Aorschungsstelle Osteuropa}

Deutsche Gesellschaft für Osteuropakunde
Otto Wolff Stiftung 


\section{Analyse}

\section{Dumawahlkampf 2007}

Jens Siegert, Moskau

\section{Zusammenfassung}

Der Ausgang der Dumawahlen ist schon heute - zwei Wochen vor der Abstimmung - weitgehend klar. Einiges Russland, massiv von der Präsidialadministration unterstützt, wird eine absolute Mehrheit erreichen. Nur die Kommunistische Partei, die in ihrer Fundamentalopposition zum System nach wie vor über Rückhalt in der Gesellschaft verfügt, wird wohl sicher in die neue Duma gelangen. Die Chancen von zwei weiteren Parteien, der ebenfalls aus dem Kreml unterstützten Partei Gerechtes Russland, die das Spektrum links der Mitte abdecken soll, und der Schirinowskij-Partei LDPR, die nationalistisch gesinnte Protestwähler anspricht, sind unklar. Den Liberalen ist es nicht gelungen, ihre Kräfte zusammenzufassen. Sie werden auch dieses Mal nicht ins Parlament einziehen. Bemerkenswert ist, dass es erstmals keine internationale Wahlbeobachtung durch die OSZE geben wird. Diese Lücke werden aber die Beobachter aus der GUS füllen, die sicher noch in der Wahlnacht eine freie, gleiche und demokratische Wahl attestieren werden.

\section{Die Dumawahl ist entschieden}

Arithmetisch ist der Ausgang der Dumawahlen am 2. Dezember 2007 längst entschieden. Die Kremlpartei Einiges Russland (ER) wird die absolute Mehrheit gewinnen. Wahrscheinlich wird dieses Ergebnis wegen der neuen Sieben-Prozent-Hürde und damit vielen Stimmen für Parteien, die daran scheitern und bei der Sitzverteilung nicht mitgezählt werden, zu einer Zweidrittelmehrheit im neuen Parlament reichen. Außer für ER lässt sich gegenwärtig nur der erneute Einzug der Kommunisten (KPRF) in das neue Parlament mit ausreichender Sicherheit voraussagen. In Meinungsumfragen erhalten sie zwischen 10 und 15 Prozent. Chancen haben noch zwei weitere bereits in der Duma vertretene Parteien. Die zweite Kremlgründung Gerechtes Russland und die Schirinowskij-Partei LDPR liegen je nach Umfrage, Umfrageinstitut und Umfragezeitpunkt mal über (eher die LDPR) und mal unter (meist das Gerechte Russland) der Sieben-Prozent-Hürde. Die liberalen Parteien Jabloko und Union der Rechten Kräfte (SPS) haben ebenso wie die weiteren fünf zur Wahl zugelassenen Parteien keine Chance in der neuen Staatsduma vertreten zu sein. Mit diesen vorausgesagten Zahlen ist aber nur wenig darüber ausgesagt, was das Ergebnis bedeutet und was daraus folgt.

\section{Parteiengesetz und Wahlgesetz}

Die Vorbereitungen des Kremls zu den diesjährigen Dumawahlen begannen schon im Sommer 2003, also noch vor der Wahl zum jetzigen Parlament. Bereits damals wurde die der Mindestanteil der Stimmen, die notwendig waren, um ins Parlament zu gelangen, für 2007 auf sieben Prozent angehoben. Begründet wurde die- se im internationalen Vergleich hohe Barriere mit dem Ziel, einen Anreiz für die nach Meinung des Kremls zu vielen kleinen Parteien zu schaffen, sich zu größeren Einheiten zusammen zu schließen. Nur so könne ein stabileres Mehrparteiensystem entstehen. Ähnlich wurden auch weitere Verschärfungen sowohl des Wahlals auch des Parteienrechts im Laufe der jetzt zu Ende gehenden Legislaturperiode begründet. Alle zielen darauf ab, die Gründung von Parteien, ihre Weiterexistenz und ihre Zulassung zu Wahlen zu erschweren und das Verhalten der Abgeordneten im Parlament zu disziplinieren. Die einschneidenste Änderung des Wahlgesetzes war die Abschaffung der Direktwahlkreise zugunsten eines reinen Verhältniswahlrechts. Während bisher analog zum deutschen Wahlrecht die Hälfte der Abgeordneten in Direktwahlkreisen gewählt wurde, wird die neue Duma nur noch über Parteilisten nach einem reinen Verhältniswahlrecht bestimmt. Unabhängige Abgeordnete wird es im neuen Parlament auch später, zum Beispiel als Folge von Partei- oder Fraktionsaustritten, nicht mehr geben. Abgeordnete, die ihre Fraktion oder Partei verlassen, verlieren künftig ihr Mandat.

Die Änderungen des Parteiengesetzes sind kaum weniger bedeutend. Neuzugründende oder bestehende Parteien müssen mindestens 50.000 Mitglieder nachweisen. Außerdem müssen in mindestens der Hälfte der gegenwärtig 86 russischen Regionen regionale Untergliederungen mit jeweils mindestens 500 Mitgliedern aktiv sein. Nur 16 registrierte Parteien konnten nach Ansicht der staatlichen Registrierungsbehörde Rosregistrazija diesen Nachweis erbringen. Alle anderen Parteien wurden im Januar dieses Jahres zwangsweise aufgelöst. Darunter befand sich auch die Russi- 
sche Republikanische Partei (RPR) des Abgeordneten Wladimir Ryschkow, der seit 1993 viermal hintereinander im Gebiet Altay ein Direktmandat erringen konnte. Ryschkow klagte gegen die Entscheidung der Registrierungsbehörde, die knapp 20.000 der von seiner Partei vorgelegten 63.000 Unterschriften für ungültig erklärte. Mitte des Jahres bestätigte das Oberste Gericht Russlands die Auflösung der Partei. Ryschkow reichte daraufhin Klage beim Europäischen Menschenrechtsgerichtshof in Straßburg ein.

\section{Die liberalen Parteien}

2003 war es keiner liberalen Partei gelungen, die damals geltende Fünf-Prozent-Hürde zu überwinden und in die Duma einzuziehen. Die gesamte Legislaturperiode über, aber mit wachsender Intensität seit Anfang 2006, wurde in unterschiedlichen Kombinationen über Zusammenschlüsse und Koalitionen liberaler Parteien und Politiker verhandelt. Grundsätzlich herrschte Einigkeit, dass es dem Kreml schwerer fallen würde, einer einigen liberalen Opposition den Wiedereinzug ins Parlament zu verwehren. Diese mehrfach und in unterschiedlichen Kombinationen geführten Verhandlungen und Auseinandersetzungen im Detail nachzuzeichnen ist hier nicht genug Raum. Die entscheidenden Grundlinien und das Ergebnis sollen aber kurz skizziert werden. Es gab fünf Hauptakteure: Jabloko, SPS, Ryschkow mit der Republikanischen Partei, den ehemaligen Ministerpräsident Michail Kasjanow und den ehemaligen Schachweltmeister Garri Kasparow. Kasparows und Kasjanows Bemühungen waren von Anfang an stärker auf die Präsidentenwahlen im kommenden Frühjahr orientiert als auf die Dumawahlen. Beide haben keine Parteien. Diese possessive Formulierung ist nicht zufällig gewählt. In ihr drückt sich das grundsätzliche Dilemma russischer Parteien aus, meist als Führerorganisationen gegründet zu werden und zu funktionieren.

Entsprechend versuchte Michail Kasjanow 2005 eine lange bestehende Partei, die Demokratische Partei, als Vorsitzender zu übernehmen. Der Versuch wurde durch eine Intrige des Kremls vereitelt und Kasjanow blieb ohne Partei. Er gründete daraufhin eine »Bewegung« unter dem Namen "Volks-Demokratische Union«, die er seit Sommer 2007 in eine Partei umzuwandeln versucht. Eine Teilnahme an der Dumawahl 2007 ist aber nicht mehr das Ziel. Garri Kasparow war 2006 der wichtigste Initiator des außerparlamentarischen Oppositionsbündnisses »Das andere Russland«. Weitere Gründer waren die National-Bolschewistische Partei (NBP) des Schriftstellers Eduard Limonow, Michail Kasjanow, der radikale Jugendverband AKM (Avantgarde der Kommunistischen Jugend) und eine Reihe weiterer Einzelpersonen. Auch Wladimir Ryschkow ge- hörte dem »Anderen Russland« zeitweise an. Insbesondere die NBP hat, was nicht nur Kasparow, sondern den meisten Oppositionsorganisationen weitgehend fehlt: eine relativ große Zahl (Schätzungen reichen von 4.000 bis 30.000) disziplinierter und aktionsbereiter, meist jugendlicher Mitglieder. Das »Andere Russland « kritisiert die Politik des Kremls grundsätzlich. Kasparow nennt das gegenwärtige politische System eine Diktatur, und schließt eine Teilnahme an Wahlen als gegenwärtig sinnlos aus, weil deren Ergebnis von der Machtelite vorbestimmt sei. "Anderes Russland « rief ab Frühjahr 2007 in verschiedenen russischen Städten zu »Märschen der Nichteinverstandenen" auf, die von massiven Polizeiaufgeboten mehrfach gewaltsam aufgelöst wurden. Jabloko und SPS schlossen sich dem »Anderen Russland « vor allem wegen der Beteiligung von nationalistischen (NBP) und stalinistischen Organisationen (u.a. AKM) nicht an. Kasparow schmähte sie daraufhin als vom Kreml gesteuert.

Verhandlungen zwischen Jabloko, SPS und Ryschkow über ein Zusammengehen oder einen Zusammenschluss scheiterten mehrfach. Die Gründe dafür sind vielfältig. Es gibt persönliche Unvereinbarkeiten, die noch aus den 1990er Jahren stammen. Platzhirschgebaren der jeweiligen offiziellen und inoffiziellen Führungspersonen hat ebenso eine Rolle gespielt wie das nicht unerhebliche Beharrungsvermögen der Parteiapparate. Der Jablokovorsitzende Grigorij Jawlinskij weist zudem darauf hin, dass man beim Zusammenschluss von zwei oder mehr Parteien nicht einfach die Umfrageergebnisse addieren könne. Viele Wähler von Jabloko seien nicht bereit für SPS zu stimmen und umgekehrt. Wie sie sich bei einem Zusammenschluss verhalten würden, sei schwer vorauszusagen. Tatsächlich gehören Jawlinskij und seine Wähler zu den langjährigen Kritikern der wilden Privatisierung der 1990er Jahre und vertreten weniger marktliberale und stärker sozial orientierte Positionen als die SPS.

Neben den angesichts der politischen Bedingungen wohl überwindbaren inhaltlichen Differenzen spielen aber auch praktische und rechtliche Probleme eine Rolle. Listenverbindungen mehrerer Parteien, die früher erlaubt waren, sind inzwischen verboten. Die Verschmelzung von zwei Parteien wäre nur durch die Gründung einer neuen dritten Partei möglich, in die die Mitglieder der sich vereinigenden Parteien jeweils individuell eintreten müssten oder dadurch, dass die Mitglieder eine Partei aus ihrer Partei austreten und in die andere Partei eintreten, weil das Gesetz die Mitgliedschaft in zwei Parteien gleichzeitig verbietet. Ersteres Szenarium hätte das große Risiko mit sich gebracht, dass die neue Partei die enormen Registrierungshürden nicht genommen hätte. Außerdem kann wohl keine der beiden Par- 
teiführungen sicher sein, dass ihr ihre gegenwärtigen Parteimitglieder auch in eine neuzugründende vereinigte Partei folgen würden. Bei letzterem Szenarium würde eine Vereinigung von Gleichberechtigten unmöglich. Eine Partei bliebe bestehen, während sich die andere auflösen müsste. Eine psychologisch sehr hohe Hürde. Trotz intensiver Vermittlungsversuche unter anderem von führenden NGO-Persönlichkeiten sind letztlich alle Vereinigungsverhandlungen gescheitert. SPS und Jabloko treten getrennt zu den Dumawahlen an. Nach dem Verbot seiner Partei verhandelte Wladimir Ryschkow mit Jabloko und SPS darüber, auf einer ihrer Listen zu kandidieren. Mit Jabloko kam es zu keiner Einigung. SPS machte Ryschkow erst das Angebot, zur sogenannten Troika, den drei Spitzenkandidaten zu gehören, zog die Offerte aber später, wohl auf Druck aus dem Kreml, wieder zurück. Umfragen aller Meinungsforschungsinstitute zeigen Jabloko und SPS weit unter der Sieben-Prozent-Hürde, ohne Chancen auf einen Parlamentseinzug.

\section{Die Kremlparteien Einiges Russland, Gerechtes Russland und Zivile Kraft}

Die Partei Einiges Russland (ER) hat bereits in der gegenwärtigen Staatsduma, trotzdem sie nur 37,5 Prozent der Stimmen bekam, eine Zwei-Drittel-Mehrheit. Zum einen gewannen ihre Kandidaten sehr viele Direktwahlkreise, zum anderen wurden unabhängige Kandidaten angeworben und Mitglieder anderer Fraktionen abgeworben. Zwischen den Wahlen sank die Popularität von ER stark und wuchs erst mit Beginn dieses Jahres und wiederholten Äußerungen Präsident Putins wieder, diese Partei stehe ihm, ob wohl er nicht Mitglied sei, am nächsten. Putin bezeichnete eine Mehrheit von ER zudem als notwendige Bedingung, damit er als Präsident seinen politischen Kurs durchsetzen könne. Inhaltliche Aussagen und das Parteiprogramm spielen dabei, wenn überhaupt, nur ein untergeordnete Rolle. Entsprechend tritt ER zu den Wahlen mit dem Slogan auf, man wolle »Putins Plan« unterstützen. Das Wahlprogramm passt auf einige wenige Seiten und ruft Putin zum "nationalen Führer» aus, dessen Rückhalt ER sei. Eine Broschüre der Partei mit dem Titel »Putins Plan« fasst lediglich mehrere Reden Putins zusammen, darunter die Ansprachen "Zur Lage der Nation« der vergangenen drei Jahre und die Rede Putins vor der Münchner Sicherheitskonferenz im Februar diesen Jahres. Entsprechend groß ist die vielleicht gar nicht unwillkommene Verwirrung bei den Wählern. Nach einer Umfrage des LewadaZentrums sind zwar 65 Prozent der Befragten davon überzeugt, dass Putin einen sorgsam ausgearbeiteten Plan zur Entwicklung des Landes hat. Aber gleichzei- tig haben 47 Prozent noch nie etwas von diesem Plan gehört und nur 6 Prozent gaben an, sie könnten erklären, was dieser Plan enthalte.

Die zweite Kremlpartei Gerechtes Russland bezeichnet sich selbst als "linkszentristisch". Herausragende programmatische Aussage ist wie bei ER die Unterstützung Putins. Im Gegensatz zu ER werden aber deutlich sozialere, ja fast schon sozialistischen Akzente vor allem in der Wirtschafts- und Sozialpolitik gesetzt. Gerechtes Russland wurde Anfang des Jahres aus der "Leben-Partei« des Föderationsratsvorsitzenden Sergej Mironow, der Rentnerpartei und den Resten der Partei Heimat gebildet. Über die Ziele dieser Gründung gibt es unterschiedliche Angaben. Wahrscheinlich handelte es sich um den neuesten Versuch, die Kommunisten zu schwächen. Zudem erfordert das vom Kreml angestrebte Zwei- oder Mehrparteiensystem einen lenkbaren »linskzentristischen « Ausgleich zum sich als »rechtszentristisch" positionierenden ER. Gerüchten zufolge soll Gerechtes Russland auf die Initiative einer der den sogenannten Silowiki, also den aus den Sicherheitsapparaten stammenden Hardlinern zugerechneten Kremlgruppen zurückgehen, die keine Zugang zu ER haben. In Umfragen wuchs der Stimmenanteil von Gerechtes Russland bei Umfragen bis zum Sommer 2007 tatsächlich langsam aber stetig bis in den zweistelligen Bereich. Die Partei schloss fast mit dem der Kommunisten auf, die gleichzeitig leicht verloren.

Im Frühsommer 2007 tauchte noch ein drittes Parteiprojekt des Kremls auf. Die vom bekannten Juristen Michail Barschtschewskij, bevollmächtigter Vertreter der russischen Regierung bei den obersten Gerichten des Landes, gegründete Organisation nannte sich anfangs Liberales Russland, wurde aber bald in Zivile Kraft umbenannt (russisch "Graschdanskaja Sila", wobei das Attribut auch bei "Zivilgesellschaft«, russisch »Graschdanskoje Obschtschestwo«, zum Einsatz kommt). Parteifarbe ist ein sattes grün, Parteisymbol die Sonnenblume. Ein Schelm ist, wer sich dabei etwas denkt. Zivile Kraft gelang es immerhin in extrem kurzer Zeit, sowohl die hohen Hürden des Parteiengesetzes als auch des Wahlgesetzes zu überwinden, sich als Partei zu konstituieren und zu den Wahlen zugelassen zu werden. Beides spricht für Unterstützung von weit oben und die damit zusammenhängende Verfügung über nicht unerhebliche Geldmittel. In Umfragen erklärten trotzdem nie mehr als ein Prozent der Befragten, für Zivile Kraft stimmen zu wollen.

\section{Kommunisten und LDPR}

Die Kommunisten bleiben, trotz der erneut im Wahljahr vom Kreml verstärkten Bemühungen über linksorientierte Parteigründungen zumindest einen Teil der 


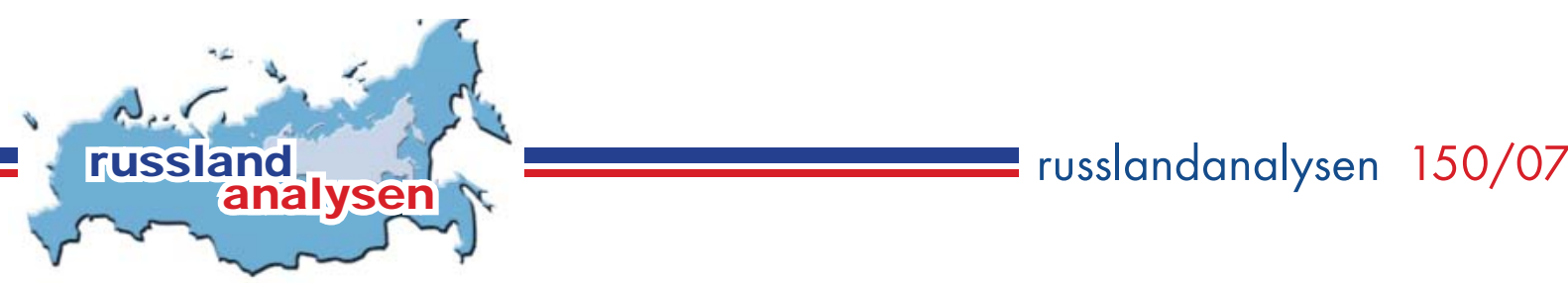

kommunistischen Wähler abzuwerben, die größte und stabilste Oppositionspartei. Wie schon 2003 die Partei Heimat, so scheint auch das Gerechte Russland eher potentielle ER-Wähler als KPRF-Wähler anzusprechen. Das ist angesichts der uneingeschränkten Unterstützung für Putin durch Gerechtes Russland auch nicht weiter verwunderlich. KPRF-Wähler verstehen sich meist als Systemoppositionelle, die den marktwirtschaftlichen Kurs Putins, ob nun mehr oder weniger sozial abgefedert, grundsätzlich ablehnen. Wahrscheinlich wird dieses Beharrungsvermögen erneut mit mehr als 10 Prozent der Stimmen belohnt werden.

Ähnlich überlebensfähig zeigt sich die Liberaldemokratische Partei Russlands (LDPR) mit ihrem Vorsitzenden Schirinowskij. Vielfach war nach der letzten Wahl ihr Niedergang vorausgesagt worden, da sie aus Kremlsicht nicht mehr benötigt werde. Die LDPR wird vorwiegend von nationalistisch gesinnten Protestwählern gewählt. Gleichzeitig stimmten und stimmen ihre Abgeordneten, man sagt gegen gute Bezahlung, stets kremlkonform und sicherten so bis 2003 der Regierung eine Mehrheit jenseits der Kommunisten. Mit der Zweidrittelmehrheit von ER ist das nicht mehr nötig. Für Schirinowskij ist Ressentiment gegen alles und jeden Programm. Es sind immer "die Anderen" (Reichen, Fremden, Beamten), die »uns" (den einfachen Russen, dem großen russischen Volk, den hier Lebenden) etwas weggenommen haben, das "eigentlich « uns gehört. Diese mit gehörigem komödiantischem Talent vorgetragenen Anklagen bringen die LDPR bei Umfragen in die Nähe oder sogar knapp über die SiebenProzent-Hürde. Für die LDPR zu stimmen, ist eine Möglichkeit, es denen »oben« zu zeigen, ohne wirklich etwas zu riskieren.

\section{Über den Autor}

Jens Siegert ist Leiter des Länderbüros Russland der Heinrich Böll Stiftung in Moskau. Zuvor arbeitete er als zehn Jahre in Moskau als Korrespondent deutschsprachiger Printmedien und Radio.

\section{Lesetipps}

- Wahlen in Russland. Deutsche Bank Research, 13. November 2007 http://www.dbresearch.de/PROD/DBR_INTERNET_DE-PROD/PROD0000000000217768.pdf

- Yevgeny Volk: Who's Who in Russia's Parliamentary Elections. WebMemo No. 1706, November 20, 2007. Published by The Heritage Foundation http://www.heritage.org/Research/RussiaandEurasia/upload/wm_1706.pdf

- Pro et Contra Journal, Volume 11, 2007 , No 4-5, July-October (Title: »Successor’s Burden« / in Russian) http://www.carnegie.ru/en/pubs/procontra/

\begin{abstract}
Keine Wahlbeobachtung durch die OSZE
Erstmals seit dem Ende der Sowjetunion wird es bei Dumawahlen keine Wahlbeobachtung der OSZE geben. Lange konnten sich die OSZE und das russische Außenministerium nicht auf die Bedingungen einer Beobachtermission und die Zahl der internationalen Wahlbeobachter einigen. Hauptstreitpunkt war die Forderung der russischen Seite, die Wahlmission zu verpflichten, vor der Verkündigung des amtlichen Endergebnisses keine öffentlichen Erklärungen und Bewertungen abzugeben. Vor vier Jahren hatte es zu großer Verstimmung im Kreml geführt, als OSZE-Vertreter schon wenige Tage nach der Wahl ihr Urteil, die Wahlen seien "frei aber nicht fair" gewesen, abgegeben hatten. Letztendlich eiman sich auf eine Kurzwahlbeobachtung und 70 brhebliche Verzögerungen bei der ViNovsteng fur die Beobachter nahm die OSZEMitte mission ganz abzusagen. Die Mission hätte angesichts der kurzen Zeit und der kleinen Anzahl von Wahlbeobachtern aber ohnehin große Probleme gehabt, Wahlfälschungen nachzuweisen. Die entscheidenden Manipulationen betreffen den Zugang zur Wahl und den Zugang zu den Massenmedien und haben längst stattgefunden. Eine Wahlbeobachtung durch die OSZE hätte kaum mehr als ein Feigenblatt für die russische Staatsführung werden können. Dieses Feigenblatt werden nun Wahlbeobachter aus den GUS-Staaten bilden. Sie werden, das ist angesichts von Erfahrungen in anderen GUS-Staaten unschwer vorauszusagen, noch in der Wahlnacht eine freie, gleiche und demokratische Wahl attestieren.
\end{abstract}




\section{Tabellen und Grafiken zum Text}

Welche folgender Parteien würden Sie bei den Wahlen für die Staatsduma Russlands wählen, wenn sie nächsten Sonntag stattfinden würden?

Quelle: Umfrage des WZIOM vom November 2007,

http://wciom.ru/novosti/press-vypuski/press-vypusk/single/9165.html

http://wciom.ru/novosti/press-vypuski/press-vypusk/single/9202.html

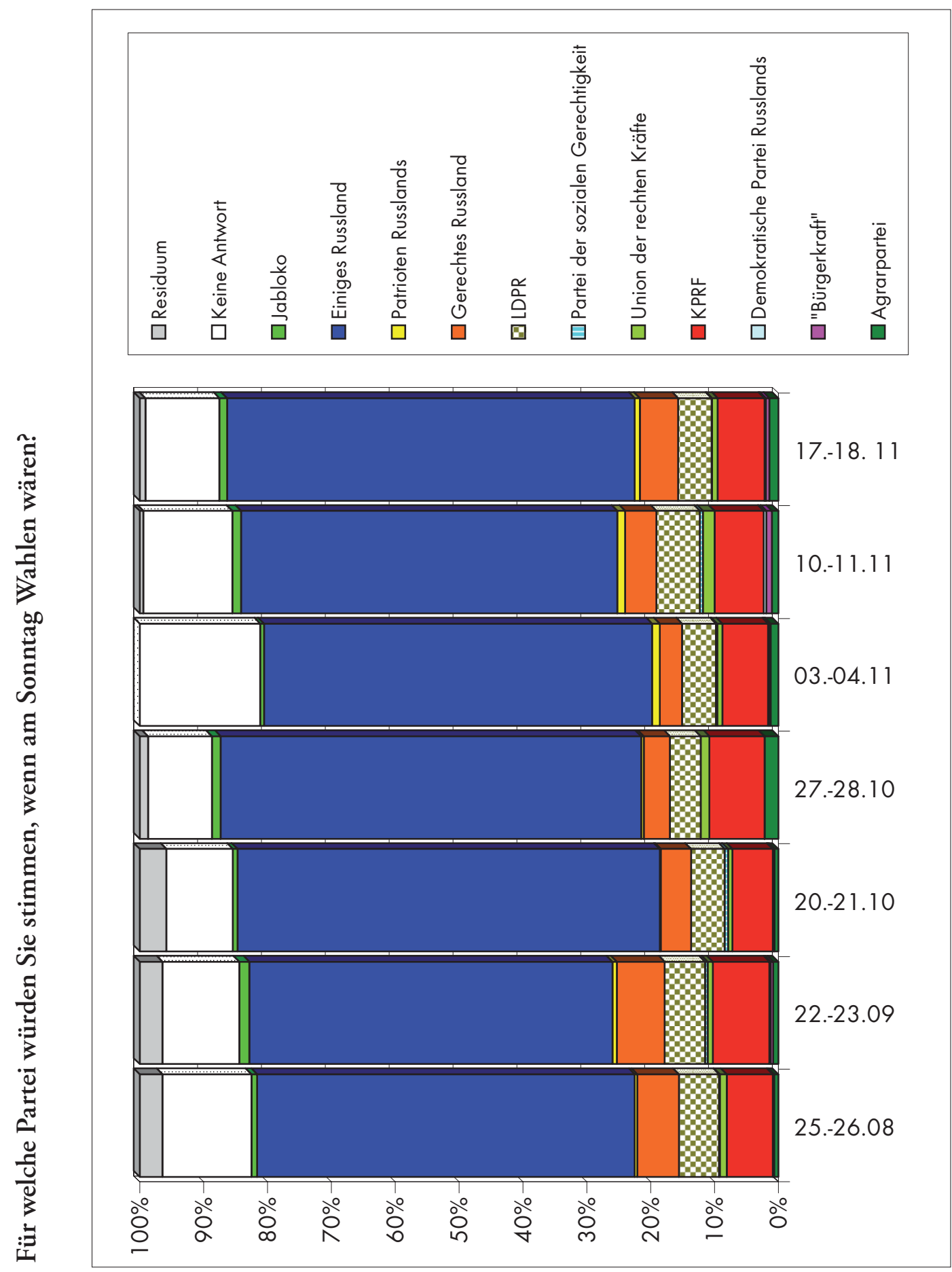





\section{Diskussionen über die Wahlen}

Quelle: Umfragen des Lewada-Zentrums vom 9.-13.November $2007 \mathrm{http}$ //www.levada.ru/files/1195047517.doc

Werden die Dumawahlen im Dezember in der Familie, unter Bekannten und Freunden besprochen?

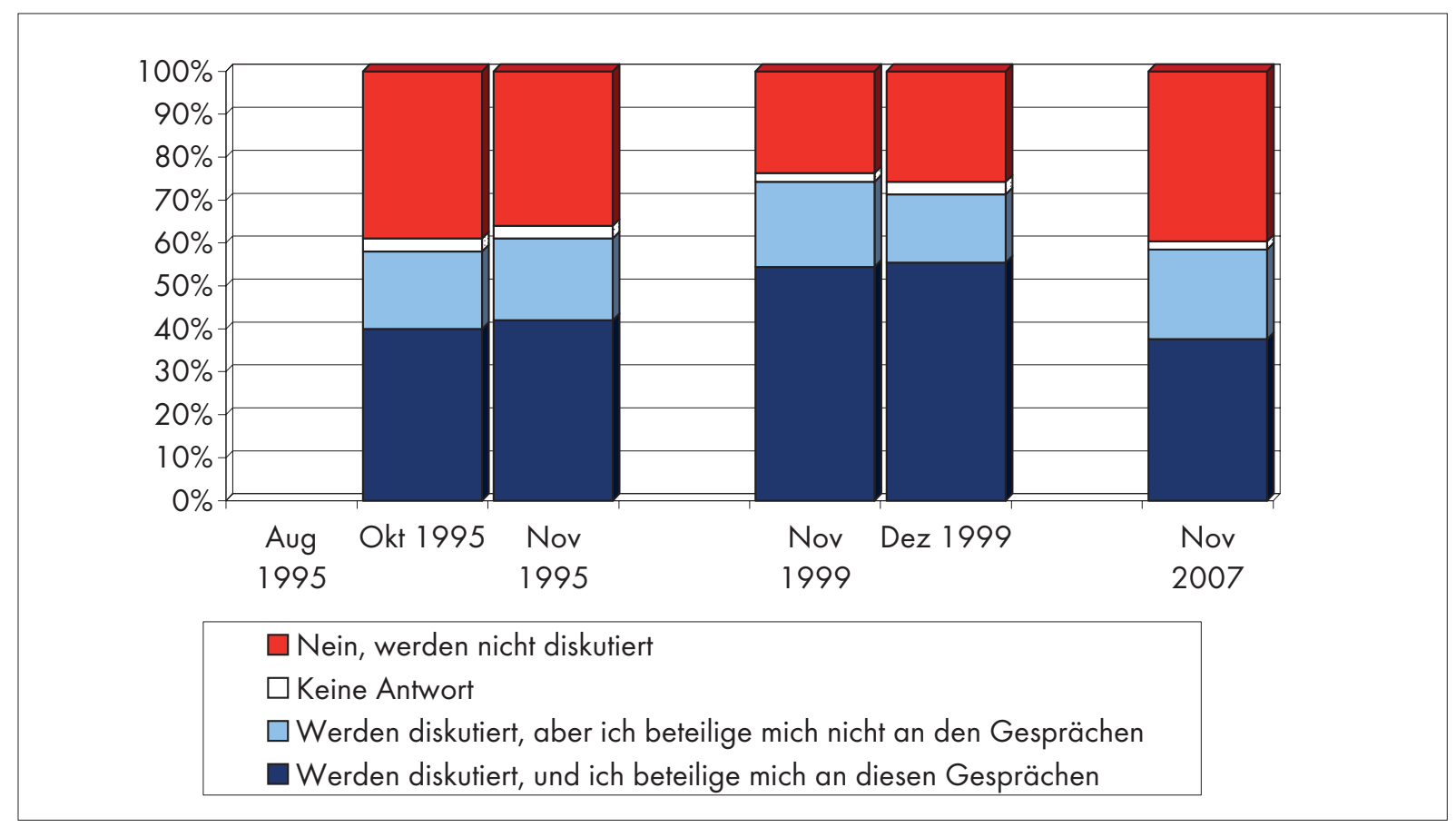

Wenn im öffentlichen Raum (Bus, Bahn etc.) ein Gespräch über die Wahlen geführt wird, würden Sie sich beteiligen?

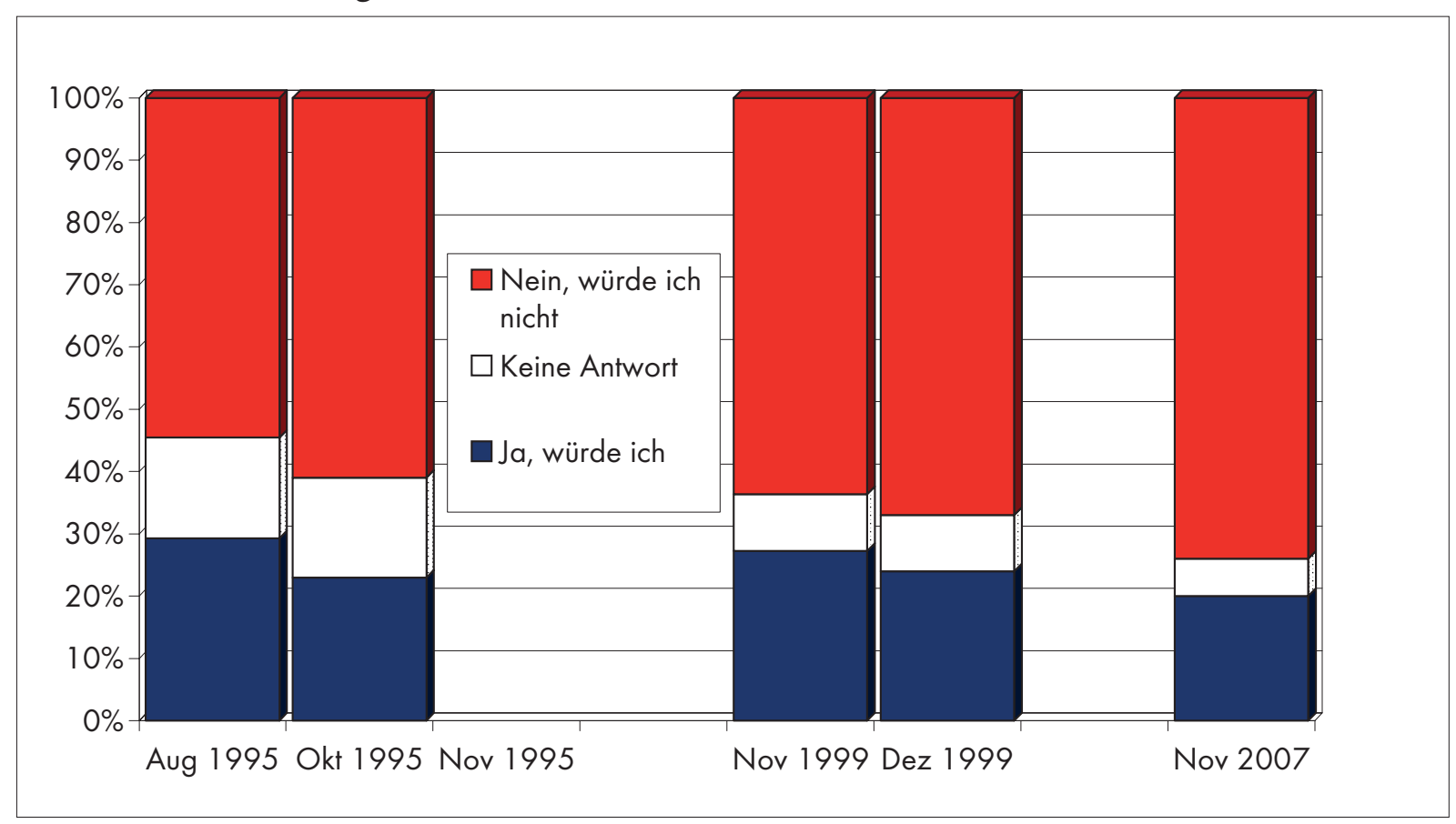




\begin{tabular}{c|c|c|c|c|c} 
August & Oktober & November & November & Dezember & November \\
1995 & 1995 & 1995 & 1999 & 1999 & 2007
\end{tabular}

Werden die Fragen, die die bevorstehenden Wahlen für die Staatsduma betreffen, in Ihrer Familie, unter Ihren Nachbarn, Arbeitskollegen diskutiert, und wenn ja, nehmen Sie an diesen Gesprächen teil?

\begin{tabular}{l|l|l|l|l|l}
$\begin{array}{l}\text { Werden diskutiert, und ich } \\
\text { beteilige mich an diesen } \\
\text { Gesprächen }\end{array}$ & $40 \%$ & $42 \%$ & $55 \%$ & $56 \%$ & $38 \%$ \\
$\begin{array}{l}\text { Werden diskutiert, aber ich } \\
\text { beteilige mich nicht an den } \\
\text { Gesprächen }\end{array}$ & $18 \%$ & $19 \%$ & $20 \%$ & $16 \%$ & $21 \%$ \\
$\begin{array}{l}\text { Nein, werden nicht diskutiert } \\
\text { Keine Antwort }\end{array}$ & $39 \%$ & $36 \%$ & $24 \%$ & $26 \%$ & $40 \%$ \\
\hline
\end{tabular}

Wenn ein Gespräch über die bevorstehenden Wahlen an einem öffentlichen Ort, z. B. in einer Warteschlange, in einem Bus, in einem Zug geführt wird, würden Sie sich in das Gespräch einschalten, würden Sie ihren Gesichtspunkt vertreten?

\begin{tabular}{|l|l|l|l|l|l|}
\hline Ja, würde ich & $29 \%$ & $23 \%$ & $27 \%$ & $24 \%$ & $20 \%$ \\
\hline Nein, würde ich nicht & $54 \%$ & $61 \%$ & $63 \%$ & $67 \%$ & $74 \%$ \\
Keine Antwort & $16 \%$ & $16 \%$ & $9 \%$ & $9 \%$ & $6 \%$
\end{tabular}

\section{Im Vorfeld der Wahlen ...}

Quelle: Umfragen des Lewada-Zentrums vom 9.-13. November 2007 http://www.levada.ru./press/2007111600.html

Was glauben Sie, wird bei den Wahlen für die Staatsduma im Jahre 2007 ein realer Kampf der Parteien um die Macht und Sitze in der Duma geführt oder wird es nur eine Vortäuschung dieses Kampfes und die Verteilung der Sitze in der Duma wird von der Macht bestimmt?

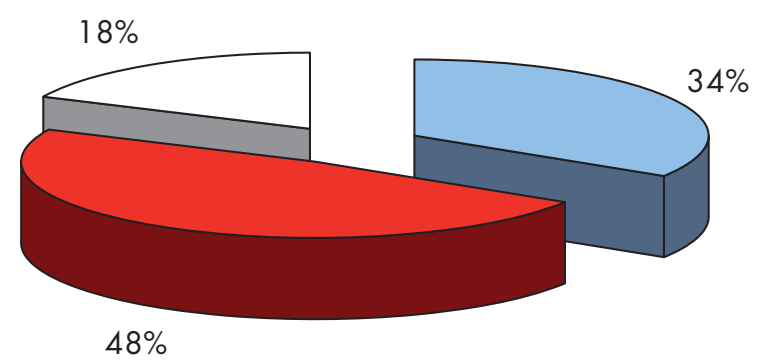

$\square$ es wird ein realer Kampf um die Macht und Sitze in der Duma geführt

$\square$ es wird nur eine Vortäuschung des Kampfes, und die Verteilung der Sitze in der Duma wird von der Macht bestimmt

$\square$ Keine Antwort 
Wählen Sie eine Eigenschaft, die Ihrer Meinung nach zu einer Partei am besten passt...

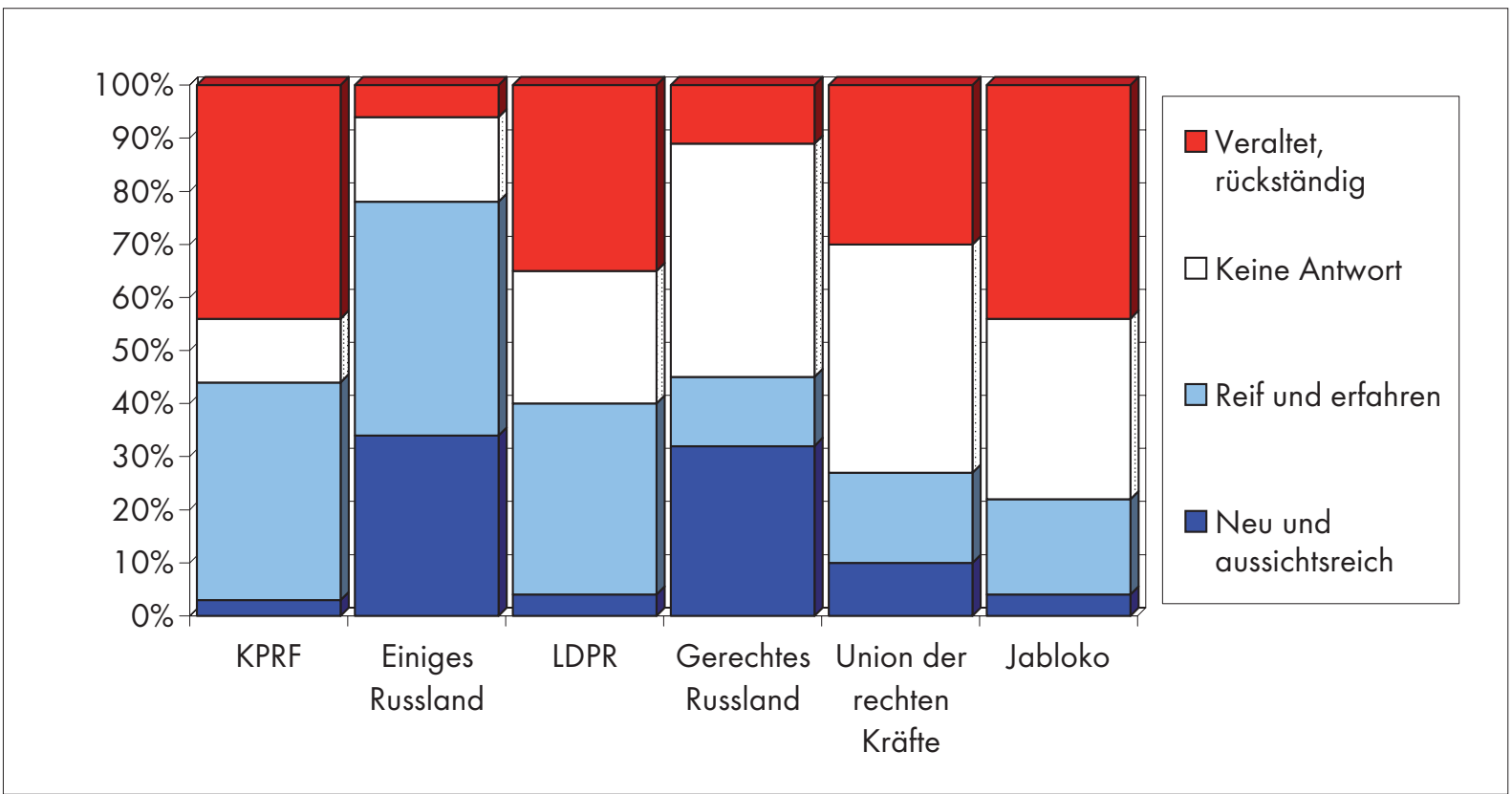

Das Bild der Parteien

\begin{tabular}{l|c|l|l|c|c} 
KPRF & $\begin{array}{c}\text { Einiges } \\
\text { Russland }\end{array}$ & LDPR & $\begin{array}{c}\text { Gerechtes } \\
\text { Russland }\end{array}$ & $\begin{array}{c}\text { Union der } \\
\text { rechten } \\
\text { Kräfte }\end{array}$ & Jabloko
\end{tabular}

Wählen Sie eine Eigenschaft, die Ihrer Meinung nach zu einer Partei am besten passt...

\begin{tabular}{l|r|r|r|r|r|r|} 
Neu und aussichtsreich & $3 \%$ & $34 \%$ & $4 \%$ & $32 \%$ & $10 \%$ & $4 \%$ \\
Reif und erfahren & $41 \%$ & $44 \%$ & $36 \%$ & $13 \%$ & $17 \%$ & $18 \%$ \\
Veraltet, rückständig & $44 \%$ & $6 \%$ & $35 \%$ & $11 \%$ & $30 \%$ & $44 \%$ \\
Keine Antwort & $12 \%$ & $16 \%$ & $25 \%$ & $44 \%$ & $43 \%$ & $34 \%$
\end{tabular}

Was glauben Sie, hat die Partei neue Ideen entwickelt?

\begin{tabular}{|l|l|l|l|l|l|l|}
\hline Hat entwickelt & $27 \%$ & $67 \%$ & $27 \%$ & $32 \%$ & $17 \%$ & $12 \%$ \\
\hline Hat keine entwickelt & $48 \%$ & $15 \%$ & $43 \%$ & $29 \%$ & $40 \%$ & $48 \%$ \\
Keine Antwort & $25 \%$ & $18 \%$ & $30 \%$ & $39 \%$ & $43 \%$ & $40 \%$ \\
\hline
\end{tabular}

Was glauben Sie, hat die Partei reale Taten, Lösungen für das Land wichtiger Probleme vollbracht?

\begin{tabular}{|l|c|c|c|c|c|c|}
\hline Hat vollbracht & $30 \%$ & $71 \%$ & $25 \%$ & $21 \%$ & $12 \%$ & $10 \%$ \\
\hline Hat keine vollbracht & $41 \%$ & $11 \%$ & $43 \%$ & $37 \%$ & $44 \%$ & $50 \%$ \\
\hline Keine Antwort & $29 \%$ & $18 \%$ & $32 \%$ & $42 \%$ & $44 \%$ & $40 \%$ \\
\hline
\end{tabular}




\section{Fernsehdebatten}

Haben Sie in den letzten zehn Tagen irgendwelche Wahlkampfdebatten der Parteiführer im Fernsehen gesehen?

\begin{tabular}{l|c} 
Ja & $29 \%$ \\
Nein & $64 \%$ \\
\hline Keine Antwort & $7 \%$
\end{tabular}

Glauben Sie, dass die Teilnahme an Fernsehdebatten für alle Parteien, die an den Wahlen teilnehmen, obligatorisch sein soll, oder glauben Sie, dass die Teilnahme der Parteien an Fernsehdebatten nicht notwendig ist, weil es reine Zeitverschwendung ist?

Die Teilnahme an Fernsehdebatten soll für alle Parteien obligatorisch sein

Die Teilnahme der Parteien an Fernsehdebatten ist nicht notwendig, weil es reine Zeitverschwendung ist

keine Antwort

$36 \%$

$44 \%$

$20 \%$

Was glauben Sie, warum „Einiges Russland“ eine Teilnahme an Fernsehdebatten abgelehnt hat?

Ihre Führer haben nichts zu sagen/ Es gibt kein positives Programm

Ihre Führer können nicht diskutieren/fürchten, sich einer offenen Polemik auszusetzen

Ihre Führer versuchen einer offenen Kritik an ihre Adresse auszuweichen

Ihre Führer wollen sich nicht auf einen Skandal und Intrigen einlassen

Ihre Führer haben keine gleichgestellten Partner, Opponenten

Diese Debatten sind sinnlos/ sie entschlossen sich, das Geld der Wähler zu sparen

Anderes

Keine Antwort

\begin{tabular}{|r|}
$6 \%$ \\
$4 \%$ \\
$13 \%$ \\
$15 \%$ \\
$15 \%$ \\
$21 \%$ \\
$5 \%$ \\
$29 \%$
\end{tabular}

\section{Dokumentation}

\section{Die "Junge Garde von `Einiges Russland« in Barnaul verunglimpft Portraits von Oppositionellen}

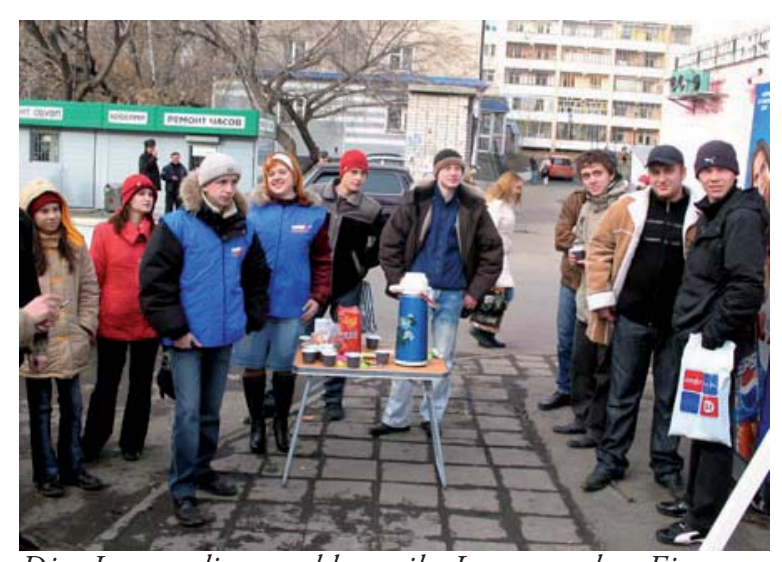

Die "Junggardisten « schlagen ihr Lager vor dem Eingang des Filmklubs der Barnauler Veteranen auf, gegen den sie regelmäßig Provokationen verüben.
Die »Junge Garde von `Einiges Russland « veranstaltete eine öffentliche Verunglimpfung der Portraits von Oppositionellen vor einem Filmklubs der Veteranen, auf den zwei Aktivisten einen Überfall verübten, für den sie vor kurzem auch verurteilt worden sind.

Ihre Aktion führte die Abteilung der JGER (Junge Garde von "Einiges Russland ") in der Altai-Region durch, als sich Barnauler Senioren anlässlich des Jahrestags der bolschewistischen Revolution von 1917 auf dem zentralen Platz der Stadt versammelten.

"Am 7. November - als Gegengewicht zu den leidig gewordenen Demonstrationen der Kommunisten - führten Aktivisten der "Jungen Garde« der Altai-Region eine Aktion "Politdarts" am Filmtheater "Premiere« durch, das jedem bekannt ist, der sich für Politik interessiert,« berichtet der 


\section{Kommentar}

\section{Nachfolgeproblem und Dumawahl}

Jens Siegert, Moskau

$\mathrm{V}$ on Anfang an stand der Dumawahlkampf unter dem Eindruck der weit wichtigeren Entscheidung über Putins Nachfolge im Frühjahr 2008. Putin hat in den vergangenen Jahren immer wieder erklärt, er werde sich an die Verfassung halten, die ihm eine erneute Kandidatur verbietet. Er lehnte es auch wiederholt ab, für eine dritte Amtszeit die Verfassung zu ändern, obwohl er angesichts der Mehrheitsverhältnisse im Parlament die Möglichkeit dazu hätte. Damit steht er im Widerspruch zu einem großen Teil der politischen Elite und auch der russischen Bevölkerung. Diese Haltung führte schon früh zu Spekulationen, wen Putin dem Volk als seinen Nachfolger vorschlagen würde und was er selbst nach der Wahl machen werde.

Im Laufe des Jahres neigten politische Beobachter immer stärker zu der Annahme, Putin werde zwar seinen Posten als Präsident tatsächlich verlassen, im Hintergrund aber weiter eine wichtige politische Rolle spielen und eventuell zu einem späteren Zeitpunkt und damit verfassungskonform erneut das Präsidentenamt anstreben. Die zahlreichen und spekulativen Modelle, wie er das zu bewerkstelligen gedenke, lassen sich in drei große Kategorien einteilen: 1. Putin wird Premierminister und die Verfassung wird so geändert, dass aus dem präsidialen ein parlamentarisches System wird; 2 . Putin empfiehlt dem Volk einen zuverlässigen Vertrauten, der für eine Übergangszeit Präsident wird, dann zurücktritt und den Weg für eine verfassungskonforme erneute Kandidatur Putins frei macht; 3. Für Putin wird ein neues Amt geschaffen, in dem er, etwa Deng Hsiao Ping gleich, mehr qua persönlicher Autorität als durch formale Befugnisse weiter das Land lenkt.

Am 1. Oktober 2007 erklärte Putin auf dem Parteitag von "Einiges Russland « für die meisten Beobachter überraschend, er nehme das Angebot der Partei an, die Liste zur Dumawahl anzuführen. Er könne sich durchaus vorstellen, Premierminister zu werden, sagte Putin außerdem. Voraussetzung dafür sei ein Wahlsieg von "Einiges Russland " und die Wahl eines "anständigen, handlungsfähigen, effektiven und modernen Menschen« zum nächsten Präsidenten. Eine Änderung der Verfassung schloss Putin erneut kategorisch aus. Durch diesen Schritt sollte der Wahlsieg für »Einiges Russland « dank Putins überragender Popularität gesichert werden. Gleichzeitig erhielte Putin, wie er selbst später öffentlich mehrfach betonte, im Falle eines hohen Wahlsiegs von »Einiges Russland« das »moralische
Recht« auch nach seiner Amtszeit als Präsident eine führende Rolle in der russischen Politik zu spielen. Tatsächlich schien diese Strategie anfangs auch aufzugehen. In ersten Umfragen nach Putins Ankündigung, Spitzenkandidat von "Einiges Russland" zu werden, stieg sowohl die Zustimmung zu ihm als auch zur Partei. Außer »Einiges Russland « und den Kommunisten schaffte es keine Partei mehr die Sieben-Prozent-Hürde zu überwinden. Die LDPR und das Gerechte Russland kamen auf 4-5 Prozent, für keine andere Partei wollten mehr als ein Prozent der Befragten stimmen. Dieser Trend hielt bis Ende Oktober und drehte sich dann um.

Dafür scheint es vor allem zwei Gründe zu geben. Zum einen durchbrach Putins Spitzenkandidatur das von ihm in seiner gesamten Amtszeit durchaus virtuos genutzte und den Menschen in Russland seit den Zeiten des russischen Imperiums und auch in der Sowjetunion wohlbekannte Schema des guten Zaren und der schlechten Bojaren. Putin steht, wie eine Art "Vater der Nation«, über der Alltagspolitik. Er gab, immer und immer wieder im Fernsehen inszeniert, den guten Führer, der sich, im Namen des Volkes und für das Volk, mit einer Schar böser, gerissener, unfähiger und räuberischer Minister, Beamter, Unternehmer und Gouverneure herumschlagen muss. Immer waren die anderen Schuld, weil sie seine Vorgaben nicht erfüllen konnten oder nicht erfüllen wollten. Erfolge wurden von der großen, aus dem Kreml gesteuerten Propagandamaschine dem Präsidenten zugeschrieben, Misserfolge den anderen. Die nun so plötzliche hergestellte Einheit von gutem Zar und bösen Bojaren überfordert aber offenbar die Wähler. Wenn der Zar sich zu den Bojaren gesellt, muss irgend etwas nicht stimmen. Das erzeugt Verwirrung anstelle des erhofften Vertrauens. Eine wachsende Zahl Wähler verweigert inzwischen die Zustimmung.

Dieser Effekt wird, und das ist der zweite Grund, von stark steigenden Preisen vor allem bei Lebensmitteln und anderen Waren, die zur Grundversorgung gehören und täglich von den Menschen gekauft werden, begleitet. Die Inflation beträgt in diesem Jahr voraussichtlich anstatt der angestrebten sieben mehr als zehn Prozent. Im Gegensatz zum oft virtuellen Spiel in der gelenkten Demokratie, in dem Dinge nach Wunsch der Regisseure oft ganz schnell erscheinen und wieder verschwinden oder ihre Gestalt verändern, sind die Preiserhöhungen und die Inflation so real wie der Pu- 
tins Aufstieg begleitende wirtschaftliche Aufschwung der vergangenen acht Jahre. Für die Preiserhöhungen sehen Wirtschaftsexperten vor allem zwei Gründe und die sind beide von der russischen Regierung zumindest kurzfristig kaum zu beeinflussen. Zum einen wich die Regierung nach dem faktischen Scheitern der Reform der nichtmonetären Sozialleistungen am unerwartet starken Protest der Bürger Anfang 2005 von ihrer bis dahin restriktiven Ausgabenpolitik ab. Politische Probleme werden seither, auch mit Blick auf die anstehenden Wahlen, großzügig mit Geld zugedeckt, an dem es ja angesichts der Hausse der Energiepreise auf dem Weltmarkt nicht mangelt. Zum anderen ist die russische Wirtschaft inzwischen viel enger mit der Weltwirtschaft verflochten als noch vor einigen Jahren. Preiserhöhungen auf dem Weltmarkt für Milchprodukte, Getreide und Energieträger, die aktuell auch in der EU spürbar sind, gehen am russischen Markt nicht mehr spurlos vorüber.

Der Kreml reagierte schnell auf den Misserfolg von Putins »Einiges Russland«-Spitzenkandidatur. Wladimir Putin kritisierte »Einiges Russland « bei einem von allen Nachrichtensendungen der landesweiten Fernsehkanäle als Aufmacher gesendeten Treffen mit Straßenbauarbeitern in einer Baubude bei Krasnojarsk am 13. November scharf. Die Partei habe viele Fehler und bis heute keine feste politische Ideologie, so Putin. Sie ziehe, wie alle Strukturen nahe der Macht, zudem jede Menge Gauner an. Trotzdem, versuchte Putin die Volte, unterstütze er diese Partei, weil es leider keine bessere gebe in Russland. Eine Stimme für "Einiges Russland « sei deshalb auch eine Stimme des Vertrauens zu ihm. So hört sich normalerweise kein Wahlkämpfer an.

Die Probleme mit Putins Spitzenkandidatur werfen die Frage auf, warum sich Putin zu diesem Schritt entschlossen hat. Eine nicht unbedeutende Rolle dürften die Auseinandersetzungen im Kreml um die Putin-Nachfolge spielen. Wer Präsident wird, hat weitreichende Auswirkungen auf das Machtgleichgewicht innerhalb der herrschenden Elite. Damit eng verbunden sind Fragen der Kontrolle über wirtschaftliche und politische Machtinstrumente, aber auch der persönlichen Sicherheit der Beteiligten. Bisher ist Putin als Person Garant der inneren Stabilität der Machtverhältnisse. Loyalität zum Präsidenten sichert die Beteiligten vor dem ganz tiefen Fall ab. Im Kreml ist im Spätsommer der Machtkampf zumindest zwischen zwei der fünf bis acht zu identifizierenden Machtgruppen offen ausgebrochen. Am 9.10.2007 erschien in der Tageszeitung Kommersant ein Artikel des Leiters der russischen Drogenbehörde Wiktor Tscherkessow. Tscherkessow, KGB-Karriereoffizier und Putin wohl auch persönlich nahestehend, warnte öffentlich vor einem »Krieg von Gruppen innerhalb der Geheimdienste«. Hintergrund war die Verhaftung mehrerer hochgestellter Beamter seiner Behörde, darunter einer im Generalsrang, durch das im vorigen Jahr neu geschaffene Untersuchungskomitee der Generalstaatsanwaltschaft. Bei der Auseinandersetzung geht es um Millionengeschäfte von Angehörigen unterschiedlicher Behörden, darunter aus dem Geheimdienst und dem Zoll. Beispiellos ist, dass diese Auseinandersetzung in die Öffentlichkeit getragen wurde. Das kann zweierlei bedeuten: Zum einen, dass der Hausherr, also Putin, nicht mehr die Macht und die Fähigkeit hat, einen derartigen Streit intern zu lösen. Zum anderen kann es eine Drohung einer internen Machtgruppe sein, die im Kampf um die Putinnachfolge zu unterliegen droht, dass sie diese Niederlage nicht einfach so hinzunehmen bereit ist. So oder so gibt es einen Vorgeschmack darauf sein, was droht, sollte Putin tatsächlich gehen. Die wichtige Botschaft ist, dass die Operation Nachfolge sehr riskant ist.

Diese machtinternen Auseinandersetzungen dürften auch eine Rolle bei der Entscheidung Putin gespielt haben, die Spitzenkandidatur von »Einiges Russland» anzustreben. Den unterschiedlichen Machtgruppen zeigte Putin so, dass er zwar zumindest vorübergehend als Präsident geht, im Hintergrund aber weiter als Schiedsrichter und Garant des Gleichgewichts bleibt, dass sich unter ihm herausgebildet hat.

Die angespannte Situation hat zu großer Nervosität im Kreml, aber auch auf allen anderen Ebenen der sogenannten Machtvertikale geführt. Die Gouverneure wurden aus dem Kreml angewiesen, beim Preis ihrer Posten, eine hohe Wahlbeteiligung, vor allem aber einen hohen Sieg von »Einiges Russland « zu liefern. Das führte zu administrativen Angriffen an allen Fronten. Das russische Internet ist voll von Berichten darüber, wie regionale Behörden Druck auf Beschäftige von staatlichen Einrichtungen und Betrieben, aber auch von Privatunternehmen ausüben. Vielen Menschen wird offenbar mit beruflichen oder sozialen Nachteilen oder dem Verlust des Arbeitsplatzes gedroht, um sie zum Eintritt bei »Einiges Russland« zu bewegen und dazu, am 2. Dezember für die Kremlpartei zu stimmen. Unter dem Eindruck des öffentlichen Unmuts über diesen den meisten Menschen in Russland noch wohlbekannten massiven administrativen Druck warnte Gleb Pawlowskij, einer der einflussreichsten Polittechnologen, zwei Wochen vor der Wahl in seiner allsamstagabendlichen Fernsehsendung "Realpolitik« davor, solche Methoden würden von »Feinden Putins«, von einer "fünften Kolonne« angewandt, die der Reputation des Präsidenten schadeten.

Die Auseinandersetzungen um die Nachfolge $\mathrm{Pu}$ tins drängen die Inhalte des Wahlkampfs weiter in den Hintergrund. Die staatliche Fernsehsender sind gesetzlich verpflichtet sind, den zur Wahl zugelassenen Par- 
teien in den letzten vier Wochen vor der Wahl, der offiziellen Wahlkampfperiode, Prime-Time-Sendezeit zur Verfügung zu stellen. Sie tun dies morgens, am frühen Abend und am 22.50 Uhr bis kurz nach Mitternacht. Protest gegen diese unattraktiven Sendezeiten wiesen die Verantwortlichen mit dem zynischen Hinweis ab, dass das erstens "Prime-Time" sei und zweitens die $\mathrm{Zu}$ schauer sich ohnehin nicht sonderlich für die Wahlsendungen interessierten. »Einiges Russland « weigerte sich, wie auch schon vor vier Jahren mit der Begründung, man spiele in einer anderen, höheren Klasse, an den Fernsehdebatten teilzunehmen. Über die Partei wird allerdings auch so oft genug in Nachrichtensendungen und politischen Magazinen berichtet und ihre Vertreter zu Interviews und Diskussionen eingeladen. Außerdem ist der "Einiges Russland «-Spitzenkandidat im russischen Fernsehen allgegenwärtig.

\section{Dokumentation}

\section{Das ODIHR sieht sich nicht in der Lage, die russischen Dumawahlen zu beobachten}

Warschau, 16. November 2007

Das OSZE-Büro für demokratische Institutionen und Menschenrechte (ODIHR) [OSCE Office for Democratic Institutions and Human Rights] hat den russischen Behörden mitgeteilt, dass das Büro nicht im Stande sein wird, ihrer Einladung, die Wahlen für die russische Staatsduma am 2. Dezember 2007 zu beobachten, zu folgen.

In einem Brief an die russische Zentrale Wahlkommission bedauerte der ODIHR-Direktor Christian Strohal diesen Ausgang, sagte jedoch, dass das ODIHR aufgrund von Verzögerungen und Restriktionen nicht in der Lage sein werde, sein Mandat wahrzunehmen.

Trotz einer verspäteten Einladung zur Beobachtung der Wahlen durch die russischen Behörden und trotz außerordentlicher Einschränkungen, die in der Einladung formuliert wurden, schickte sich das ODIHR daran, sein Mandat gewissenhaft und in gutem Glauben wahrzunehmen, indem es sich bemühte, trotz der vielen Einschränkungen, die der Institution auferlegt worden waren, eine Beobachtungsmission zu entsenden.

Das ODIHR beantragte, 20 Experten am 7. November und weitere 50 Beobachter am 15. November entsenden zu können. Trotz wiederholter Versuche, für Experten und Beobachter des ODIHR Einreisevisa in die Russische Föderation zu erhalten, sind Einreisevisa kontinuierlich verweigert worden.

Deshalb schließt das ODIHR daraus, dass die Behörden der Russischen Föderation nicht willens sind, ODIHRBeobachter rechtzeitig und auf kooperative Weise zu empfangen und mit ihnen uneingeschränkt zusammenzuarbeiten. Daher erklärt das ODIHR zu seinem Bedauern, dass es sich nicht in der Lage sieht, sein Mandat unter diesen Umständen wahrzunehmen.

Presseerklärung des OSCE Office for Democratic Institutions and Human Rights (ODIHR) vom 16.11.2007

Quelle: http://www.osce.org/odihr/item_1_27967.html

\section{Pressestimmen}

\section{Pressestimmen zur Absage der Wahlbeobachtung durch das ODIHR}

\section{Spiegel (Hamburg), 19.11.2007}

\section{OSZE weist russische Vorwürfe zurück}

"Es ist bedauerlich, dass uns keine andere Wahl gelassen wurde«, sagte der oberste Wahlbeobachter der Organisation für Sicherheit und Zusammenarbeit in Europa (OSZE), Christian Strohal, in einem Reuters-Interview. [...]

Zudem warf das Außenministerium in Moskau Strohal indirekt vor, sich von den USA instrumentalisieren zu lassen. „Dieser Vorwurf entbehrt jeglicher Grundlage«, sagte Strohal dazu. Es sei reiner Zufall gewesen, dass er kurz vor der Absage der Russland-Mission eine Rede in Washington gehalten habe. 
Es sei sehr ungewöhnlich, dass Russland die Visa-Erteilung für die Wahlbeobachter so lange hinausgezögert habe, sagte Strohal als Leiter des OSZE-Büros für demokratische Institutionen und Menschenrechte (ODIHR). Die OSZE entsende jedes Jahr zahlreiche Mitarbeiter in verschiedene Länder, ohne dass es jemals Visa-Probleme gebe. "So etwas ist uns noch nie passiert«, erklärte Strohal mit Blick auf das Verhalten der russischen Behörden. Es bleibe zu hoffen, dass sich das Problem bei der Präsidentenwahl im nächsten Jahr nicht wiederhole.

Quelle: http://www.spiegel.de/politik/ausland/0,1518,518260,00.html

Neue Zürcher Zeitung (Zürich), 16.11.2007

Keine Wahlbeobachtung der OSZE in Russland

[...] Das offizielle Russland reagierte gelassen und verlogen zugleich auf die Entscheidung des ODIHR. Es sei dessen gutes Recht, so zu handeln, beschied der Sprecher des Moskauer Außenministeriums süffisant. Doch hieß es in einer scharfen Erklärung der Behörde, der Grund dafür liege einzig im Chaos, das im ODIHR herrsche. Die Probleme seien vorgeschoben. Igor Borisow, ein Mitglied der Wahlkommission, kehrte den Spieß um und warf der Institution vor, nicht einmal ihre grundlegenden Verpflichtungen erfüllen zu vermögen. Das sei der letzte Beweis für die Notwendigkeit der Reform des Büros. [...]

Quelle: http://www.nzz.ch/nachrichten/international/russland_wahlen_osze_1.585521.html

Kommersant (Moskau), 21.11.2007

Dumawahlen bleiben nicht ohne Aufmerksamkeit internationaler Beobachter (Michail Sygar u. a.)

[...] Die internationalen Beobachter haben gestern bezüglich der Dumawahlen eine endgültige Entscheidung getroffen. Es werden alle kommen, außer den Experten des Büros für demokratische Institutionen und Menschenrechte. Die Opposition setzt nur auf ihre Beobachter und glaubt, dass die Anwesenheit ausländischer Experten die Wahlen weder ehrlicher noch freier machen werde. [...]

"Wir sind bis jetzt alle zufrieden«, sagte der Leiter des Departments der Parlamentarischen Versammlung des Europarates für zwischenparlamentarische Zusammenarbeit und Wahlbeobachtung Wladimir Dronow. [...]

Der offizielle Vertreter der Parlamentarischen Versammlung der OSZE, Klas Bergman, versicherte dem Kommersant nur, dass auf jeden Fall Beobachter kommen und unbedingt ein ehrliches und unabhängiges Monitoring der Wahlen durchführen werden. [...]

Quelle: http://www.kommersant.ru/doc.aspx? DocslD=827770\&Nodes ID =2

Komsomolskaja prawda (Moskau), 21.11.2007

Dmitrij Medwedew: Die Absage der OSZE ihre Beobachter zu entsenden ist entweder ein Fehler oder eine geplante Aktion (Faina Keller)

Der erste stellvertretende Premier der RF glaubt, dass die Entscheidung des ODIHR, ihre Beobachter nicht zu den Wahlen am 2. Dezember zu entsenden, ein Fehler ist. »Es ist eine merkwürdige Fehlposition, die aussieht, als wäre sie im Voraus so geplant gewesen.« [...] »Ich möchte keinen verdächtigen, aber unsere europäischen Kollegen schwiegen, obwohl man ihnen erklärt hatte, wie und wo man Visa bekommt, und verkündeten ihre Position erst nach einer Dienstreise in die USA", bemerkte Medwedew. "Es ist möglich, dass es nur ein Zufall ist." Laut Medwedew ist Russland offen für die weitere Zusammenarbeit mit der OSZE.

Quelle: http://www.kp.ru/online/news/38745/ 


\section{Chronik}

\section{Vom 14. bis zum 22. November 2007}

\begin{tabular}{|c|c|}
\hline 14.11 .2007 & $\begin{array}{l}\text { Der Leiter der inguschetischen Transportpolizei, Oberst Salman Arapchanow, wird in seinem Haus in Ordshonikid- } \\
\text { sewskaja von Unbekannten erschossen. }\end{array}$ \\
\hline $\begin{array}{l}14 . / \\
15.11 .2007\end{array}$ & $\begin{array}{l}\text { ie russische Flugaufsicht verweigert am 14.11. einer Bundeswehrmaschine auf dem Weg nach Termes (Usbekistan) die } \\
\text { Tutzung des russischen Luftraums. Am 15.11. wird der Überflug wieder gestattet. }\end{array}$ \\
\hline 15.11 .2007 & $\begin{array}{l}\text { In Twer eröffnet ein regionaler »Für Putin!«-Kongress. Ausländischen Korrespondenten und den Journalisten bestimm- } \\
\text { ter russische Medien wird eine Akkreditierung »aus Platzgründen« verweigert. Der Kongress fordert Präsident Putin } \\
\text { auf, im Amt zu bleiben. }\end{array}$ \\
\hline 15.11 .2007 & Die Stadtverwaltung von Moskau verbietet eine Demonstration, die die Bewegung »Anderes Russland« für den 24.11. plant. \\
\hline 15.11 .2007 & $\begin{array}{l}\text { Das Katastrophenschutzministerium teilt mit, dass der Ölteppich in der Straße von Kertsch am 16.11. das Asowsche } \\
\text { Meer erreichen wird. }\end{array}$ \\
\hline 15.11 .2007 & $\begin{array}{l}\text { Der Stellvertretende Finanzminister Sergej Stortschak wird unter dem Verdacht der Korruption festgenommen. Offenbar } \\
\text { ist in den Fall, bei dem es um die Rückzahlung sowjetischer Schulden geht, auch eine Reihe von Banken involviert. }\end{array}$ \\
\hline 15.11 .2007 & $\begin{array}{l}\text { Sicherheitskräfte stürmen ein Haus in Stepnoj (südlich von Machatschkala, Dagestan) und töten zwei Personen, die dem } \\
\text { Untergrund zugerechnet werden. }\end{array}$ \\
\hline 15.11 .2007 & $\begin{array}{l}\text { Bei einem Schusswechsel auf der Autobahn Machatschkala-Kaspijsk werden zwei Personen getötet, die als Angehöriger } \\
\text { illegaler bewaffneter Formationen identifiziert werden. }\end{array}$ \\
\hline 16.11 .2007 & $\begin{array}{l}\text { Der Föderationsrat bestätigt einstimmig ein Gesetz, das Russlands Beteiligung am Vertrag über Konventionelle Streit- } \\
\text { kräfte in Europa (KSE) aussetzt. }\end{array}$ \\
\hline 16.11 .2007 & ( \\
\hline 17.11.2007 & n Ulluby Balatchanow, der wegen der Ermordung meh- \\
\hline 19.11 .2007 & $\begin{array}{l}\text { Die USA und Russland unterzeichnen ein Abkommen, das die Beseitigung von } 34 \mathrm{t} \text { waffenfähigem Plutonium auf rus- } \\
\text { sischer Seite vorsieht. }\end{array}$ \\
\hline 19.11 .2007 & Bei einem Feuergefecht mit Unbekannten werden in Naltschik ein Polizist getötet und zwei weitere verwundet. \\
\hline 20.11 .2007 & $\begin{array}{l}\text { In seiner Residenz Nowo Ogarowo empfängt Präsident Putin Saad Hariri, den Vorsitzenden der Mehrheitsfraktion des } \\
\text { libanesischen Parlaments. }\end{array}$ \\
\hline 20.11 .2007 & $\begin{array}{l}\text { Michail Kamynin, der Sprecher des russischen Außenministeriums erklärt, dass der Boykott der Wahlen in Kosovo durch } \\
\text { die serbische Minderheit, die Legitimität dieser Wahlen in Frage stellt. }\end{array}$ \\
\hline 20.11 .2007 & Die SPS wird zum wiederholten Maße Opfer von IT(Hacker)-Attacken. \\
\hline 20.11 .2007 & $\begin{array}{l}\text { Putin spricht vor der Kommandeurstagung der russischen Streitkräfte und kritisiert die Muskelspiele der NATO an den } \\
\text { Grenzen Russlands. Putin kündigt eine Gehaltserhöhung für Militärs an und eine bessere Wohnraumversorgung. }\end{array}$ \\
\hline 20.11 .2007 & $\begin{array}{l}\text { Iwan Bolschakow, ein Dumakandidat der Partei Jabloko und führendes Mitglied der Jugendbewegung der Partei, wird } \\
\text { verhaftet, nachdem er kurz vorher bei der Zentralen Wahlkommission eine Beschwerde gegen den Fernsehsender »Ers- } \\
\text { ter Kanal« wegen illegaler Wahlkampfunterstützung für Putin und "Einiges Russland« eingebracht hatte. Vorwand für } \\
\text { seine Verhaftung war ein Zusammenstoß mit der Polizei, der einen Monat früher stattgefunden hatte. }\end{array}$ \\
\hline 20.11.2007 & $\begin{array}{l}\text { Dmitrij Muratow, der Herausgeber der Zeitung »Nowaja gaseta» erhält den »International Press Freedom Award», den } \\
\text { das New Yorker »Committee to Protect Journalists« verleiht. }\end{array}$ \\
\hline 21.11 .2007 & $\begin{array}{l}\text { Putin hält die Hauptrede auf der zentralen Wahlkampfveranstaltung von "Einiges Russland» und der Bewegung "Za } \\
\text { Putina« im Moskauer Sportkomplex "Lushniki«. Ausländische und unabhängige Medien erhalten für das Ereignis kei- } \\
\text { ne Akkreditierung }\end{array}$ \\
\hline 22.11 .2007 & $\begin{array}{l}\text { Die Inspektion der Föderalen Steuerbehörden veranlasst die Streichung des Unternehmens Jukos aus dem staatlichen } \\
\text { Register juristischer Personen. }\end{array}$ \\
\hline 22.11 .2007 & $\begin{array}{l}\text { William Bowring, Koordinator des Europäischen Zentrums für Verteidigung der Menschenrechte (EHRAC) und Be- } \\
\text { rater des britischen Entwicklungsministeriums, der in Astrachan an einem Seminar teilnahm, wird aus Russland ausge- } \\
\text { wiesen, da er nur ein Touristenvisum hat. }\end{array}$ \\
\hline 22.11 .2007 & Putin empfängt den italienischen Regierungschef Roman Prodi in Moskau. \\
\hline 22.11.2007 & $\begin{array}{l}\text { Der Spitzenkandidat der Partei Jabloko für die Staatsduma in Dagestan, Farid Babajew, wird in Machatschkala von ei- } \\
\text { nem Unbekannten angeschossen und schwer verwundet. }\end{array}$ \\
\hline
\end{tabular}

Die Russlandanalysen werden mit Unterstützung durch die Otto-Wolff-Stiftung gemeinsam von der Forschungsstelle Osteuropa an der Universität Bremen und der Deutschen Gesellschaft für Osteuropakunde herausgegeben.

Die Meinungen, die in den Russlandanalysen geäußert werden, geben ausschließlich die Auffassung der Autoren wieder.

Abdruck und sonstige publizistische Nutzung sind nach Rücksprache mit der Redaktion gestattet.

Redaktion und technische Gestaltung: Matthias Neumann, Heiko Pleines, Hans-Henning Schröder Russlandanalysen-Layout: Cengiz Kibaroglu

ISSN 1613-3390 @ 2007 by Forschungsstelle Osteuropa, Bremen

Forschungsstelle Osteuropa • Publikationsreferat • Klagenfurter Str. 3 - 28359 Bremen • Telefon: +49 421-218-7891 - Telefax: +49 421-218-3269

e-mail: publikationsrefera@@osteuropa.uni-bremen.de • Internet-Adresse: www.russlandanalysen.de 


\section{Lesehinweis}

\section{Kostenlose E-Mail-Dienste der Forschungsstelle Osteuropa}

\section{Russlandanalysen}

Die "Russlandanalysen« bieten wöchentlich eine Kurzanalyse zu einem aktuellen Thema, ergänzt um Grafiken und Tabellen. Zusätzlich gibt es eine Wochenchronik aktueller politischer Ereignisse.

Abonnement unter: fsopr@uni-bremen.de

\section{Russian Analytical Digest}

Der Russian Analytical Digest bietet zweimal monatlich englischsprachige Kurzanalysen sowie illustrierende Daten zu einem aktuellen Thema.

Abonnement unter: http://www.res.ethz.ch/analysis/rad/

\section{kultura. Russland-Kulturanalysen}

Die Russland-Kulturanalysen diskutieren in kurzen, wissenschaftlich fundierten, doch publizistisch-aufbereiteten Beiträgen signifikante Entwicklungen der Kultursphäre Russlands. Jede Ausgabe enthält zwei Analysen und einige Kurztexte bzw. Illustrationen. Erscheinungsweise: monatlich, in je einer deutschen und englischen Ausgabe.

Abonnement unter: fsopr@uni-bremen.de

\section{Ukraine-Analysen}

Die Ukraine-Analysen bieten zweimal monatliche eine Kurzanalyse zu einem aktuellen Thema aus Politik, Wirtschaft oder Kultur, ergänzt um Grafiken und Tabellen. Zusätzlich gibt es eine Chronik aktueller Ereignisse.

Abonnement unter: fsopr@uni-bremen.de

\section{Polen-Analysen}

Die Polen-Analysen bieten zweimal monatliche eine Kurzanalyse zu einem aktuellen Thema aus Politik, Wirtschaft oder Kultur, ergänzt um Grafiken und Tabellen. Zusätzlich gibt es eine Chronik aktueller Ereignisse.

Abonnement unter: http://www.polen-analysen.de

\section{Bibliographische Dienste}

Die vierteljährlich erscheinenden Bibliographien informieren über englisch- und deutschsprachige Neuerscheinungen zu Polen, Russland, Tschechischer und Slowakischer Republik sowie zur Ukraine. Erfasst werden jeweils die Themenbereiche Politik, Außenpolitik, Wirtschaft und Soziales.

Abonnement unter: fsopr@uni-bremen.de 